\title{
EL SISTEMA DE I+D EN ANDALUCÍA: UNA EVALUACIÓN DE LOS GRUPOS DE INVESTIGACIÓN
}

Jesús BASULTO SANTOS *

F.M. SOLÍS CABRERA *

\section{INTRODUCCIÓN.}

Como es bien sabido, es a partir de la Ley 13/1986 de Fomento y Coordina. ción General de la Investigación Científica y Técnica (Ley de la Ciencia) y e Plan Nacional de Investigación y Desarrollo Tecnológico cuando comienza c aplicarse una política dirigida a crear un Sistema de Ciencia y Tecnología er España.

Aquí la Ciencia debe entenderse como una acumulación de conocimientos básicos y la Tecnología como una aplicación de estos conocimientos a la produc. ción de bienes y servicios (Ranis, 1978). Aunque se tiene la creencia de que existen relaciones entre Ciencia y Tecnología, su verdadera naturaleza es difíci de percibir (Solo y Rogers, 1972; Tisdell, 1981 y Sato y Suzaova, 1983).

En el Sistema español de Ciencia y Tecnología se considera que el objetivc final del mismo debe contener elementos de bienestar económico y social tales como el crecimiento y productividad de la economía nacional y la calidad de vida (A. Lafuente y L.A. Oro, 1992).

La estructura autonómica del Estado español prevista en la Constitución hs producido un efecto notable sobre el Sistema de Ciencia y Tecnología. La mayo] parte de las Comunidades Autónomas han emprendido iniciativas para coordina] y fomentar la investigación y la tecnología en su ámbito territorial y disponen po: ello de órganos de planificación de la I+D (Investigación y Desarrollo) y d normativas específicas para tal fin. Así, por ejemplo, en la Comunidad Autónom: de Andalucía se aprueba el Plan Andaluz de Investigación (P.A.I.) en 1990, par: un período de cuatro años (1990-1993) y con un presupuesto de 18.000 millone:

*. Departamento de Economía Aplicada I. de la Universidad de Sevilla. 
de pesetas de fondos propios. Con la aprobación de este Plan culmina el proceso iniciado en 1984, cuando Andalucía asume las competencias en Educación e Investigación con la puesta en marcha del Programa de Política Científica, con una inversión de 7.983 millones de pesetas hasta el año 1989, permitió iniciar un Sistema de Ciencia y Tecnología en Andalucía. Para un conocimiento del proceso emprendido y una evaluación del Sistema puede consultarse el "Plan Andaluz de Investigación 1990-1993".

En Andalucía el Plan Nacional y el Plan Andaluz de Investigación abrieron grandes expectativas al desarrollo de la investigación y tecnología, con diversos tipos de ayudas para la formación de personal investigador, realización de Proyectos de Investigación -generalmente en líneas prioritarias- adquisición de infraestructura y otros tipos de acciones para favorecer la innovación en la empresa (por ejemplo, la creación del Instituto de Fomento Andaluz). Como muestra del interés que creó el Plan Andaluz de Investigación, recogemos la siguiente estadística sobre convocatorias de ayudas a Grupos de Investigación.

TABLA 1

\begin{tabular}{lrccc}
\hline & $1988 / 89$ & $1989 / 90$ & $1990 / 91$ & $1991 / 92$ \\
\hline № de Grupos & 797 & 933 & 1.055 & 1.227 \\
Ayudas (MPTA) & 1.492 & 1.500 & 1.527 & 1.792 \\
\hline
\end{tabular}

Fuente: Consejería de Educación y Ciencia. Junta de Andalucía

Y si consideramos las personas que forman los Grupos de Investigación, resultan las siguientes estadísticas acerca de los recursos humanos.

TABLA 2

\begin{tabular}{lrrr}
\hline & 1989 & 1990 & 1991 \\
\hline Doctores & 4.048 & 4.603 & 5.287 \\
T. Superiores (No Doct.) & 2.738 & 3.432 & 4.407 \\
Diplomados Universit. & 264 & 362 & 458 \\
Becarios & 1.102 & 1.240 & 1.406 \\
TOTAL & 8.214 & 9.650 & 11.569 \\
\hline
\end{tabular}

Fuente: Consejería de Educación y Ciencia. Junta de Andalucía 
Aunque el Plan Nacional y el Plan Andaluz de Investigación cuentan co medios para evaluar las distintas peticiones, en el inicio no se tenía experienci sobre cómo evaluar la Producción Científica y el Desarrollo Tecnológico y s conocía mal la situación de España en relación a los países de su entorno respec to de la Producción de Ciencia y Tecnología. Para poder evaluar las Política Nacionales sobre Ciencia y Tecnología fue necesario emprender estudios d Bibliometría y usar las bases de datos correspondientes (por ejemplo, el Scienc Citation Index, entre otros). Precisamente un primer trabajo que permite medj la Producción Científica con detalle de los recursos científicos asentados e diversas regiones españolas es el elaborado por Maltrás y Quintanilla (1992) Otros trabajos que traten sobre Investigación o Tecnología pueden verse en la "II Jornadas de Investigación sobre las Relaciones entre Ciencia, Tecnologíc Economía y Sociedad (1993)".

El Instituto Nacional de Estadística (INE) viene publicando, desde el bieni 1969 y 1970, estadísticas sobre los recursos destinados a actividades de Investiga ción Científica y Desarrollo Tecnológico en cada uno de los sectores, Empresas Administración Pública, Enseñanza Superior e Instituciones Privadas sin fines d lucro. A partir de 1980, el INE ha revisado las estadísticas relativas a la Enseñan za Superior como consecuencia de la encuesta sobre empleo del tiempo de profesorado universitario, realizada durante el curso 1989/90. También el INI procedió en 1987 a efectuar una regionalización de los recursos destinados a I+I por todos los sectores económicos.

El Plan Andaluz de Investigación, viene elaborando, desde su primera convo catoria, una base de datos que recoge la Producción Científica y otros tipos d variables de los Grupos de Investigación. La información contenida en la bas de datos servirá para medir la producción científica en Andalucía, así como otra características de los Grupos de Investigación (tamaño, organización, relacione: entre grupos, etc. .. ).

Nuestra experiencia en el uso de las estadísticas recogidas en la base de dato: del Plan Andaluz de Investigación (F.M. Solís Cabrera, J. Basulto Santos y C Arias Martín, 1992), nos ha conducido a elaborar un indicador de Producciór Científica (propuesto en F.M. Solís Cabrera y J. Basulto Santos, 1993) que no: permite ordenar, dentro de cada ponencia, a los Grupos de Investigación. En e presente artículo se dan algunas aplicaciones de dicho indicador así como lá producción científica por tipo de instituciones de investigación (Universidades CSIC, etc. . . ). A continuación se estudian las relaciones entre el indicador de Producción Científica y cada una de las variables: número de doctores, licencia. dos no doctores, diplomados universitarios, becarios, tamaño y financiación; estc último permite explicar la variabilidad del indicador de producción científica. 
A partir de aquí el artículo ha sido estructurado en un segundo apartado donde se recoge información sobre la investigación en Andalucía, en los apartados tres y cuatro donde se dan aplicaciones del indicador de Producción Científica y se establecen relaciones con otras variables recogidas en el Plan Andaluz de Investigación, respectivamente. Por último, en el apartado cinco, se recogen las principales conclusiones que hemos obtenido de nuestro estudio.

\section{LA INVESTIGACIÓN EN ANDALUCÍA.}

Desde hace años las Instituciones y los investigadores especializados en la gestión de la investigación y el desarrollo (I+D) se plantean el dilema de qué indicadores serán los más adecuados para medir los inputs y los outputs en el Sistema de Ciencia y Tecnología de una Comunidad.

Aunque no se ha llegado a una respuesta definitiva, en lo que respecta a los inputs, los indicadores más utilizados a la hora de medir éstos son los gastos y el personal dedicados a I+D. Respecto a los outputs, los más utilizados son la Producción Científica, los proyectos y las patentes.

En España desde que las Comunidades Autónomas van asumiendo las competencias en investigación y se van conociendo los primeros datos sobre las actividades de cada Comunidad en $\mathrm{I}+\mathrm{D}$, se observan grandes desequilibrios regionales, tanto en los inputs como en los outputs, según se pone de manifiesto en diversos estudios realizados sobre Investigación y Desarrollo (Martín y Romero, 1988; Maltrás y Quintanilla, 1992; Martín, Moreno y Romero, 1990; Acosta y Coronado, 1991). Dichos estudios han revelado también, sobre todo el de Maltrás y Quintanilla (1992), que la correlación entre los niveles de renta regional y el esfuerzo en I+D realizado en una región, no es significativa. Así mientras que Andalucía ocupaba el penúltimo lugar, en cuanto al nivel de renta per cápita, entre las 17 Comunidades Autónomas, es la cuarta Comunidad en lo que respecta a inversiones en $\mathrm{I}+\mathrm{D}$, detrás de Madrid, Cataluña y País Vasco, además, en la Comunidad de Madrid se contabilizaron 328 empresas con departamento en I+D, 738 en Cataluña, 68 en el País Vasco y únicamente 24 empresas con departamento en I+D en Andalucía (Memoria de Plan Nacional 1992). Esto último significa que en Andalucía el gran esfuerzo inverso en I+D proviene mayoritariamente del sector público.

España ha pasado de destinar el 0,64\% del P.I.B., en el año 1987, a actividades de $\mathrm{I}+\mathrm{D}$ a un $0,9 \%$ registrada en 1992 (ver las memorias del Plan Nacional). El esfuerzo relativo por Comunidad Autónoma respecto al V.A.B., sitúa Andalucía en el octavo lugar con un índice de (Gastos en I+D)/V.A.B. de 0,5. 
En cuanto a las medidas de los outputs del Sistema, según los resultado obtenidos en el reciente trabajo de Maltrás y Quintanilla (1992), sobre Producció Científica en España en la década de los años ochenta -SCI-, Andalucía ocup el tercer lugar, después de Madrid y Cataluña, en cuanto al número total d documentos publicados. La producción científica proviene fundamentalmente d las Universidades, los Centros del CSIC y los Hospitales. Las provincias d Sevilla, Granada y Córdoba producen el $90 \%$ de los documentos publicados e] Andalucía y recogidos en el SCI.

Hemos de decir que aunque la base de datos SCI es Ia más prestigiosa a nive internacional, debemos señalar que recoge los documentos publicados en una 3.200 revistas, la mayoría publicadas en lengua inglesa y con algunas laguna importantes, pues mientras las publicaciones en Biomedicina están ampliament representadas, no ocurre lo mismo con los campos de la Tecnología, Ingenieríc Informática, etc..

Con respecto al uso de las bases internacionales sobre Producción Científicধ tal como el SCI, los expertos intemacionales aconsejan que los países poc desarrollados en Ciencia deben elaborar sus propias bases de datos internacione les, ya que las bases internacionales no recogen de forma adecuada la Producció Científica de los países menos desarrollados. Conscientes de este último hech los responsables del Plan Andaluz de Investigación, viene elaborando desde s primera convocatoria una base de datos que recoge la Producción Científica otros tipos de variables de los Grupos de Investigación.

Como una muestra de los datos recogidos por el Plan Andaluz de Investigació damos a continuación la Producción Científica de los Grupos de Investigación cc rrespondientes a los tres últimos años y sobre los que se tiene información:

TABLA 3

NÚMERO DE DOCUMENTOS PUBLICADOS

\begin{tabular}{lrrr}
\hline & 1989 & 1990 & 1991 \\
\hline Revistas Internacionales & 2.249 & 2.465 & 2.664 \\
Revistas Nacionales & 3.611 & 3.630 & 2.638 \\
Congresos Internacionales & 2.217 & 2.479 & 2.838 \\
Congresos Nacionales & 3.003 & 3.468 & 3.814 \\
Tesis Doctorales & 722 & 842 & 836 \\
Patentes & 24 & 39 & 39 \\
Proyectos & 1.175 & 1.362 & 1.749 \\
Libros y Cap. de libros & 1.984 & 2.332 & 2.663 \\
\hline
\end{tabular}

Fuente: Consejería de Educación y Ciencia. Junta de Andalucía 
En el apéndice damos estadísticas, para los años 89,90 y 9 I, sobre la producción en Revistas Internacionales, Nacionales, Congresos Internacionales, Nacionales, Tesis Doctorales, Libros y Capítulos de Libros, Patentes y Proyectos y desagregados por las siete líneas prioritarias. En la siguiente tabla recogemos la Producción Científica por Doctor para los tres últimos años conocidos:

TABLA 4

DOCUMENTOS PUBLICADOS POR DOCTOR

\begin{tabular}{lccc}
\hline & 1989 & 1990 & 1991 \\
\hline Revistas Internacionales & 0,55 & 0,53 & 0,52 \\
Revistas Nacionales & 0,88 & 0,79 & 0,72 \\
Congresos Internacionales & 0,54 & 0,54 & 0,56 \\
Congresos Nacionales & 0,73 & 0,75 & 0,74 \\
Tesis Doctorales & 0,18 & 0,18 & 0,16 \\
Proyectos & 0,28 & 0,29 & 0,35 \\
Libros y Cap. de libros & 0,48 & 0,51 & 0,51 \\
\hline
\end{tabular}

Fuente: Consejería de Educación y Ciencia. Junta de Andalucía

\section{INDICADOR DE PRODUCCIÓN CIENTÍFICA DE LOS GRUPOS DE INVESTIGACIÓN.}

Con objeto de poder estudiar el comportamiento de los Grupos de Investigación respecto a su Producción Científica, hemos recogido las siguientes variables que vienen a medir distintos tipos de conocimiento generado por dichos Grupos.

- Número de trabajos publicados en Revistas Internacionales (RI).

- Número de trabajos publicados en Revista Nacionales (RN).

- Número de Comunicaciones presentadas a Congresos Internacionales (CI).

- Número de Comunicaciones presentadas a Congresos Nacionales (CN).

- Número de Tesis Doctorales Ieídas o dirigidas (TD).

- Número de Patentes (PT).

- Número de Libros o Capítulos de Libros (LC).

- Número de Proyectos realizados (PR).

Los datos corresponden a los años 89, 90 y 91 están desagregados por las siete líneas prioritarias. El total de Grupos de Investigación asciende a 1227. 
Antes de comenzar nuestro estudio, cada una de las variables ha sido converti da a una variable categórica. A partir de varias pruebas, las categorías que $\mathrm{s}$ han definido para cada una de las variables originadas son las que ha continua ción recogemos.

Para las variables RI, RN, CI y CN las categorías consideradas son: (0), [1-5. [6-10], [11-15] y $\geq 16$. Para la variable TD, sus categorías son: (1), (2), (3) y $\geq 4$ Para la variable PT, sus categorías son: (0) y $\geq 1$. Y, por último, para la variables LC y PR, las categorías consideradas son: (0), [1-5], [6-10] y $\geq 11$.

$\mathrm{El}$ motivo de convertir todas las variables a variables categóricas ha sido par. poder aplicar el Análisis de Homogeneidad (A. GIFI, 1990; J.J. Busto (1991)) $\mathrm{El}$ interés que tiene el Análisis de Homogeneidad se debe a que es muy genera y permite tratar tanto las relaciones lineales como no lineales que puedan existi entre las variables originales. Hemos aplicado el Análisis de Homogeneidar dentro de cada línea prioritaria y se ha seleccionado el primer factor que asign: una cuantificación a los Grupos de Investigación. Este primer factor ha sid denominado "Producción Científica", las razones de correlación en porcentaje entre este indicador de Producción Científica, dentro de cada línea, y cada un: de las variables categóricas vienen recogidas en la siguiente tabla:

TABLA 5

\begin{tabular}{lrrrrrrr}
\hline & P.G.C. & A.A. & C.V. & R.N. & C.S.H. & T.P. & T.I. \\
\hline R.I. & 39,89 & 58,25 & 29,91 & 36,25 & 35,05 & 63,19 & 44,44 \\
R.N. & 43,15 & 33,95 & 47,72 & 44,04 & 63,70 & 13,03 & 23,63 \\
C.l. & 50,41 & 71,85 & 45,54 & 42,51 & 48,75 & 71,95 & 53,03 \\
C.N. & 36,54 & 60,84 & 55,69 & 42,74 & 48,12 & 52,23 & 55,34 \\
T.D. & 23,88 & 57,42 & 46,46 & 46,44 & 38,50 & 45,82 & 47,81 \\
L.C. & 34,70 & 25,47 & 33,00 & 41,92 & 64,04 & 19,79 & 38,91 \\
P.T. & 9,29 & 6,21 & 0,12 & 2,35 & 0,81 & 2,82 & 22,25 \\
P.R. & 25,27 & 56,71 & 8,59 & 44,02 & 18,10 & 53,31 & 41,56 \\
\hline
\end{tabular}


En esta tabla se puede observar altas razones de correlación entre el indicador de Producción Científica y determinadas variables categóricas, dependiendo de la línea considerada.

Para poder representar el comportamiento de todos los Grupos de Investigación y para cada línea prioritaria, se ha particionado en 15 intervalos iguales los valores del indicador de Producción Científica y, a continuación, se han construido los histogramas recogidos en la tabla seis.

Es preciso aclarar que los grupos que aparecen en el primer intervalo y que por consiguiente la variable de producción toma la primera categoría son su mayor parte grupos que no han actualizado su curriculum.

Por último recogemos en la tabla siete, para la línea de Agroalimentación la relación entre las 15 categorías del indicador de producción y cada una de las variables categóricas consideradas.

Esta última tabla nos confirma lo adecuado del indicador propuesto para representar el conjunto de las variables que miden la producción científica de los grupos de investigación.

Para ayudar a la interpretación de los histogramas se debe entender que el intervalo donde aparece el valor 0 del indicador, los grupos de investigación con dicho valor o con valores situados en dicho intervalo toman los valores promedios de las variables de producción considerada. Con esta interpretación, se observa en el histograma, que en la línea prioritaria de agroalimentación, el $58,8 \%$ de los grupos están por encima del comportamiento promedio; en ciencias de la vida el porcentaje es del $40 \%$, en recursos naturales y medio ambiente dicho valor baja al 30,5\%, para ciencias sociales y humanísticas sube el 50,2\% y para las líneas de tecnologías de producción y de la información los porcentajes son del $55,8 \%$ y $34,8 \%$ respectivamente. Estos porcentajes no deben interpretarse, por ejemplo, como que hay "mejores" grupos de investigación en ciencias sociales y humanidades que en tecnología de la información, en realidad los grupos de la línea tecnología de la información están más orientados hacia las publicaciones en revistas internacionales y las comunicaciones en congresos internacionales, mientras que en los grupos de ciencias sociales y humanidades se orientan hacia revistas nacionales y congresos nacionales e internacionales, los grupos de la línea de tecnología de la información tienen que soportar mayor competencia tanto entre ellos como con investigadores de otros países, y de ahí que sea más difícil superar al grupo promedio.

Este último nos pones de manifiesto la precaución y cuidados que tenemos que tener a la hora de comparar la producción científica de una ponencia con otra. 


\section{TABLA 6}

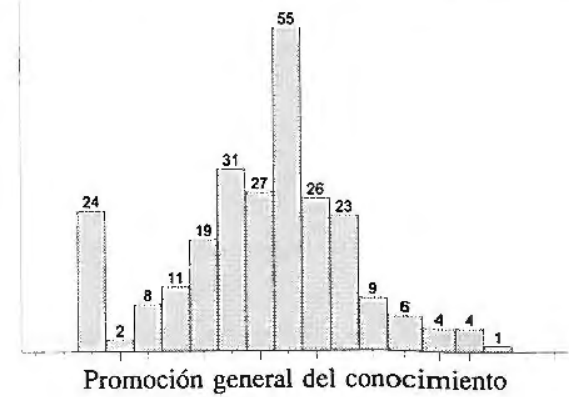

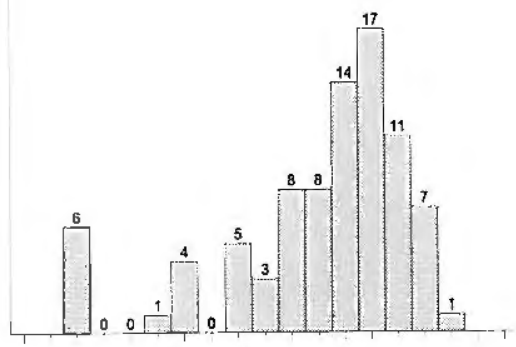

Agroalimentación

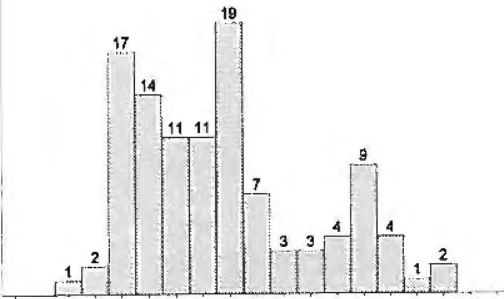

Recursos naturales y medio ambiente

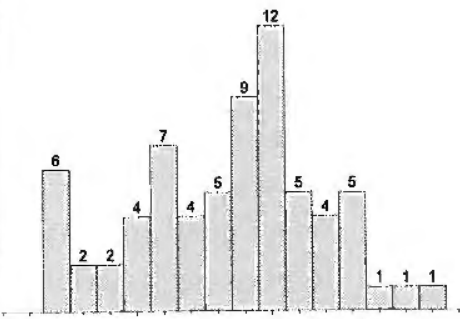

Tecnologías de Producción

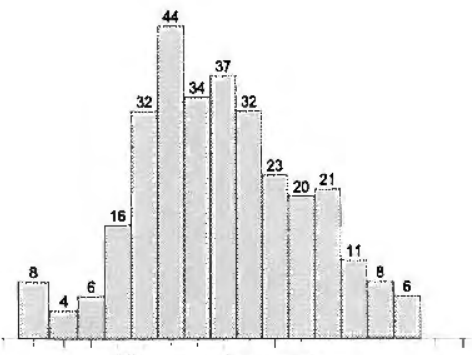

Ciencias de la vida

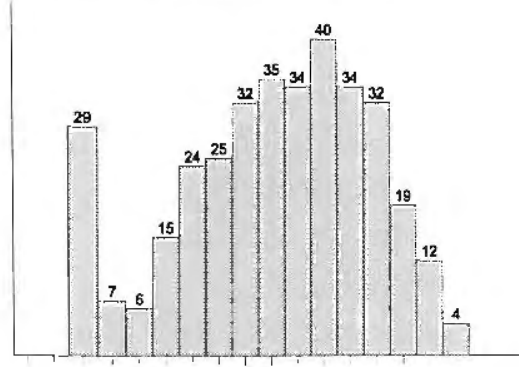

Ciencias sociales y humanísticas

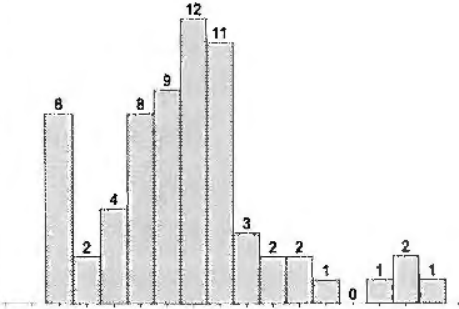

Tecnologías de la Información 
Otra aplicación del indicador de Producción Científica es el comparar los distintos organismos que desarrollan investigación dentro de cada línea prioritaria. En las tablas siguientes hemos recogido los resultados de cruzar los organismos con el indicador de Producción Científica.

TABLA 7

\begin{tabular}{|c|c|c|c|c|c|c|c|c|}
\hline \multicolumn{9}{|c|}{ PONENCIA 2} \\
\hline & $\begin{array}{c}\text { R.I. } \\
12345\end{array}$ & $\begin{array}{c}\text { R.N. } \\
12345\end{array}$ & $\begin{array}{c}\text { C.I. } \\
12345\end{array}$ & $\begin{array}{c}\text { C.N. } \\
12345\end{array}$ & $\begin{array}{c}\text { T.D. } \\
12345 \\
\end{array}$ & $\begin{array}{c}\text { L. } \\
1234\end{array}$ & $\begin{array}{r}\text { P.T. } \\
12 \\
\end{array}$ & $\begin{array}{r}\text { P.R. } \\
123 \\
\end{array}$ \\
\hline 1 & 60000 & 60000 & 60000 & 60000 & 60000 & 6000 & 60 & 6000 \\
\hline 2 & 00000 & 00000 & 00000 & 00000 & 00000 & 0000 & 00 & 0000 \\
\hline 3 & 00000 & 00000 & 00000 & 00000 & 00000 & 0000 & 00 & 0000 \\
\hline 4 & 10000 & 01000 & 10000 & 01000 & 10000 & 0100 & 10 & 1000 \\
\hline 5 & 12100 & 12100 & 21100 & 30100 & 40000 & 3100 & 40 & 2200 \\
\hline 6 & 00000 & 00000 & 00000 & 000000 & 00000 & 0000 & 00 & 0000 \\
\hline 7 & 31010 & 04100 & 32000 & 31100 & 04000 & 2210 & 50 & 1400 \\
\hline 8 & 11100 & 21000 & 03000 & 12000 & 11100 & 0300 & 30 & 1200 \\
\hline 9 & 23210 & 06011 & 05300 & 24200 & 33110 & 3401 & 71 & 1610 \\
\hline & 12230 & 33110 & 04130 & 06200 & 04301 & 5300 & 62 & 1511 \\
\hline & 05540 & 24530 & 06611 & 09221 & 26312 & 6611 & 95 & 1832 \\
\hline & 04931 & 28331 & 07325 & 08432 & 05642 & 11141 & 143 & 0764 \\
\hline & 00344 & 01631 & 01712 & 03314 & 03026 & 2630 & 110 & 0443 \\
\hline & 02104 & 00421 & 00115 & 01213 & 01015 & 0241 & 43 & 0223 \\
\hline 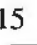 & 00001 & $\begin{array}{lllll}0 & 0 & 0 & 0 & 1\end{array}$ & 000001 & 000001 & 00001 & 0100 & 01 & 00001 \\
\hline
\end{tabular}

De la observación de estas tablas se deduce que, por ejemplo, para la línea de Ciencias de la Vida (Tabla 9), del 40\% de los Grupos que están por encima de su promedio, el $55 \%$ de ellos son de las Universidades de Sevilla (33\%) y de Granada (67\%).

En el caso de Recursos Naturales y Medio Ambiente (Tabla 10), del 30,5\% de los Grupos que están por encima de su promedio, el $52 \%$ son de la Universidad de Granada.

En Ciencias Sociales y Humanísticas (Tabla 11), del 50,2\% de los Grupos que superan su promedio, el $81 \%$ de ellos se reparten entre las Universidades de Sevilla (46\%), Málaga (21\%) y Granada (33\%). En la líneas de las Tecnologías de la Producción (Tabla 12), del 55,8\% de los Grupos con producción superior a los Grupos promedios, el 55\% de ellos son de la Universidad de Sevilla. Por último, vamos a referirnos a la línea de Agroalimentación (Tabla 8) que con $58,8 \%$ de Grupos superando su promedio, el $64 \%$ de ellos se reparten entre la Universidad de Córdoba (63\%) y el CSIC (38\%). 
TABLA 8

AGROALIMENTACIÓN

\begin{tabular}{llllllllllllllll}
\hline Otros & 2 & 0 & 0 & 0 & 0 & 0 & 0 & 0 & 1 & 0 & 1 & 2 & 1 & 1 & 0 \\
C.S.I.C. & 1 & 0 & 0 & 0 & 1 & 0 & 1 & 2 & 2 & 2 & 5 & 4 & 1 & 1 & 1 \\
U. Sevilla & 0 & 0 & 0 & 1 & 0 & 0 & 0 & 1 & 0 & 1 & 4 & 1 & 1 & 0 & 0 \\
U. Málaga & 0 & 0 & 0 & 0 & 0 & 0 & 0 & 0 & 0 & 0 & 0 & 0 & 1 & 0 & 0 \\
U. Granada & 1 & 0 & 0 & 0 & 0 & 0 & 1 & 0 & 0 & 0 & 0 & 3 & 0 & 1 & 0 \\
U. Córdoba & 2 & 0 & 0 & 0 & 2 & 0 & 3 & 0 & 5 & 5 & 3 & 6 & 7 & 4 & 0 \\
U. Cádiz & 0 & 0 & 0 & 0 & 1 & 0 & 0 & 0 & 0 & 0 & 1 & 1 & 0 & 0 & 0 \\
\hline
\end{tabular}

TABLA 9

CIENCIAS DE LA VIDA

\begin{tabular}{llllllllllllllll}
\hline Otros & 5 & 1 & 0 & 0 & 2 & 4 & 5 & 5 & 2 & 5 & 3 & 3 & 1 & 0 & 1
\end{tabular}

$\begin{array}{llllllllllllllll}\text { C.S.I.C. } & 0 & 0 & 0 & 0 & 2 & 3 & 4 & 3 & 2 & 4 & 0 & 1 & 0 & 0 & 0\end{array}$

U. Sevilla $\quad \begin{array}{llllllllllllllll} & 1 & 1 & 2 & 7 & 15 & 16 & 12 & 9 & 6 & 6 & 3 & 2 & 4 & 1 & 0\end{array}$

$\begin{array}{llllllllllllllll}\text { U. Málaga } & 0 & 2 & 2 & 1 & 1 & 4 & 2 & 5 & 2 & 2 & 0 & 5 & 0 & 0 & 0\end{array}$

$\begin{array}{llllllllllllllll}\text { U. Granada } & 0 & 0 & 0 & 3 & 7 & 8 & 7 & 10 & 12 & 7 & 7 & 5 & 3 & 5 & 5\end{array}$

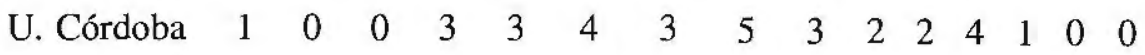

$\begin{array}{llllllllllllllll}\text { U. Cádiz } & 1 & 0 & 2 & 0 & 1 & 4 & 2 & 1 & 3 & 1 & 4 & 2 & 2 & 2 & 0\end{array}$

TABLA 10

RECURSOS NATURALES Y MEDIO AMBIENTE

\begin{tabular}{llllllllllllllll}
\hline Otros & 0 & 0 & 0 & 0 & 0 & 0 & 0 & 0 & 0 & 0 & 0 & 0 & 0 & 0 & 0 \\
C.S.I.C. & 0 & 0 & 3 & 2 & 3 & 2 & 5 & 3 & 0 & 1 & 0 & 0 & 0 & 1 & 0 \\
U. Sevilla & 0 & 1 & 4 & 2 & 3 & 2 & 4 & 0 & 1 & 0 & 1 & 2 & 0 & 0 & 0 \\
U. Málaga & 0 & 1 & 3 & 3 & 2 & 1 & 2 & 1 & 0 & 0 & 1 & 1 & 2 & 0 & 0 \\
U. Granada & 0 & 0 & 3 & 5 & 2 & 5 & 6 & 3 & 2 & 2 & 2 & 5 & 1 & 0 & 2 \\
U. Córdoba & 1 & 0 & 4 & 1 & 0 & 0 & 2 & 0 & 0 & 0 & 0 & 1 & 1 & 0 & 0 \\
U. Cádiz & 0 & 0 & 0 & 1 & 1 & 1 & 0 & 0 & 0 & 0 & 0 & 0 & 0 & 0 & 0 \\
\hline
\end{tabular}


TABLA 11

CIENCIAS SOCIALES Y HUMANÍSTICAS

\begin{tabular}{lccccccccccccccc}
\hline Otros & 2 & 0 & 0 & 0 & 0 & 2 & 0 & 0 & 2 & 0 & 1 & 0 & 0 & 0 & 0 \\
C.S.I.C. & 0 & 0 & 0 & 0 & 0 & 0 & 0 & 0 & 1 & 2 & 1 & 2 & 1 & 0 & 0 \\
U. Sevilla & 11 & 2 & 2 & 6 & 9 & 8 & 12 & 10 & 10 & 17 & 13 & 12 & 6 & 6 & 1 \\
U. Málaga & 5 & 0 & 1 & 2 & 4 & 4 & 5 & 7 & 3 & 7 & 5 & 5 & 7 & 2 & 1 \\
U. Granada & 6 & 2 & 0 & 5 & 6 & 5 & 8 & 9 & 9 & 10 & 10 & 10 & 4 & 3 & 1 \\
U. Córdoba & 3 & 2 & 1 & 0 & 2 & 2 & 4 & 5 & 6 & 4 & 4 & 3 & 1 & 1 & 1 \\
U. Cádiz & 2 & 1 & 2 & 2 & 3 & 4 & 3 & 4 & 3 & 0 & 1 & 0 & 0 & 0 & 0 \\
\hline
\end{tabular}

TABLA 12

TECNOLOGÍAS DE LA PRODUCCIÓN

\begin{tabular}{llllllllllllllll}
\hline Otros & 0 & 0 & 1 & 0 & 0 & 0 & 0 & 0 & 0 & 0 & 0 & 0 & 0 & 0 & 0
\end{tabular}

$\begin{array}{llllllllllllllll}\text { C.S.I.C. } & 0 & 0 & 0 & 0 & 0 & 0 & 0 & 0 & 0 & 1 & 0 & 1 & 0 & 0 & 1\end{array}$

$\begin{array}{llllllllllllllll}\text { U. Sevilla } & 1 & 0 & 1 & 3 & 3 & 2 & 3 & 6 & 7 & 2 & 3 & 2 & 1 & 0 & 0\end{array}$

$\begin{array}{llllllllllllllll}\text { U. Málaga } & 0 & 1 & 0 & 0 & 3 & 1 & 1 & 3 & 2 & 1 & 0 & 0 & 0 & 1 & 0\end{array}$

$\begin{array}{llllllllllllllll}\text { U. Granada } & 1 & 0 & 0 & 1 & 0 & 1 & 0 & 0 & 0 & 0 & 1 & 0 & 0 & 0 & 0\end{array}$

$\begin{array}{llllllllllllllll}\text { U. Córdoba } & 1 & 0 & 0 & 0 & 1 & 0 & 0 & 0 & 1 & 0 & 0 & 1 & 0 & 0 & 0\end{array}$

$\begin{array}{llllllllllllllll}\text { U. Cádiz } & 3 & 1 & 0 & 0 & 0 & 0 & 1 & 0 & 2 & 1 & 0 & 1 & 0 & 0 & 0\end{array}$

TABLA 13

TECNOLOGÍAS DE LA INFORMACIÓN

\begin{tabular}{llllllllllllllll}
\hline Otros & 1 & 0 & 0 & 0 & 0 & 0 & 0 & 0 & 0 & 0 & 0 & 0 & 0 & 0 & 0 \\
C.S.I.C. & 1 & 0 & 0 & 0 & 1 & 3 & 1 & 1 & 0 & 1 & 0 & 0 & 0 & 0 & 0 \\
U. Sevilla & 2 & 1 & 2 & 3 & 2 & 2 & 2 & 0 & 1 & 0 & 0 & 0 & 0 & 1 & 1 \\
U. Málaga & 0 & 0 & 0 & 1 & 2 & 2 & 4 & 1 & 0 & 1 & 1 & 0 & 0 & 0 & 0 \\
U. Granada & 2 & 1 & 1 & 2 & 2 & 4 & 3 & 0 & 1 & 0 & 0 & 0 & 1 & 1 & 0 \\
U. Córdoba & 0 & 0 & 0 & 2 & 2 & 1 & 0 & 1 & 0 & 0 & 0 & 0 & 0 & 0 & 0 \\
U. Cádiz & 2 & 0 & 1 & 0 & 0 & 0 & 1 & 0 & 0 & 0 & 0 & 0 & 0 & 0 & 0 \\
\hline
\end{tabular}


TABLA 14

PROMOCIÓN GENERAL DEL CONOCIMIENTO

\begin{tabular}{lccccccccccccccc}
\hline Otros & 1 & 0 & 0 & 0 & 0 & 0 & 0 & 0 & 0 & 0 & 0 & 0 & 0 & 0 & 0 \\
C.S.I.C. & 0 & 0 & 0 & 0 & 0 & 1 & 0 & 0 & 1 & 0 & 0 & 0 & 0 & 1 & 0 \\
U. Sevilla & 9 & 2 & 3 & 5 & 8 & 9 & 8 & 17 & 5 & 9 & 1 & 2 & 2 & 1 & 0 \\
U. Málaga & 3 & 0 & 2 & 1 & 5 & 6 & 4 & 12 & 4 & 3 & 1 & 0 & 0 & 0 & 0 \\
U. Granada & 6 & 0 & 2 & 2 & 4 & 11 & 8 & 12 & 11 & 8 & 7 & 4 & 1 & 1 & 0 \\
U. Córdoba & 1 & 0 & 0 & 1 & 1 & 2 & 3 & 5 & 4 & 1 & 0 & 0 & 0 & 1 & 1 \\
U. Cádiz & 4 & 0 & 1 & 2 & 1 & 2 & 4 & 9 & 1 & 2 & 0 & 0 & 1 & 0 & 0 \\
\hline
\end{tabular}

\section{RELACIONES ENTRE EL INDICADOR DE PRODUCCIÓN} CIENTÍFICA Y OTRAS VARIABLES RECOGIDAS POR EL P.A.I.

En este apartado se estudia las relaciones entre el indicador de Producción Científica y cada una de las siguientes variables:

- Número de Doctores (DO).

- Número de Titulados Superiores no Doctores (TS).

- Número de Diplomados (DI).

- Personal Auxiliar (PA).

- Becarios (BE).

- Ayuda Económica recibida en miles de pesetas (AE).

- Tamaño del Grupo (TG).

Todas estas variables han sido transformadas a variables categóricas y las categorías que se han definido para cada una de las variables son las que a continuación recogemos.

Para las variable DO y TS las categorías consideradas son (0), (1), [2-3], [4-6] [7-10] y $\Rightarrow>11$. Para la variable DI, las categońas son: $(0),(1), y=>2$. Para PA se ha tomado las categorías: (0), (1), (2) y >=3. Para BE, se tomó: (0), [1-2] y $=>3$. Para la variable $\mathrm{AE}$, las categorías seleccionadas han sido $(0),(0,500],(500,1000],(100$, $2000],(2000,2500]$ y $>2500$. Por último, para la variable tamaño, a partir de cuantificar cada Doctor con un punto, cada Titulado Superior no doctor con medio punto y cada Diplomado con un cuarto de punto, se ha participado en las categonas siguientes: $(0,1,5],(1,5,4,5],(4,5,8,5],(8,5,12,5],(12,5,16,5]$ y $>16,5$. 
Recogemos en la tabla siguiente los coeficientes de correlación al cuadrado (en porcentajes) entre el indicador y cada una de las variables consideradas; todos los cálculos se han hecho para cada una de las líneas prioritarias.

TABLA 15

\begin{tabular}{lccccccc}
\hline & P.G.C. & A.A. & C.V. & R.N. & C.S. & T.P. & T.I. \\
\hline Doctores & 19 & 42 & 28 & 49 & 36 & 47 & 43 \\
Licenciados & 8 & 6 & 7 & 22 & 10 & 7 & 10 \\
Diplomados & 1 & 1 & 0 & 3 & 1 & 24 & 4 \\
Auxiliares & 1 & 9 & 3 & 3 & 1 & 2 & 11 \\
Becarios & 8 & 17 & 1 & 11 & 9 & 23 & 7 \\
Ayudas recibidas & 48 & 78 & 11 & 34 & 58 & 63 & 55 \\
Tamaño & 16 & 26 & 27 & 50 & 32 & 33 & 36 \\
\hline
\end{tabular}

FUENTE: Elaboración propia

Observando la tabla, vemos que la variable más importante para explicar la Producción Científica es el número de doctores; en algunas líneas, por ejemplo, Recursos Naturales y Medio Ambiente el coeficiente de determinación es del $49 \%$. Le sigue en importancia la ayuda recibida por el Grupo y el tamaño de cada Grupo. Del resto de variables destaca el número de becarios.

En la línea de Tecnología de Producción, el modelo de regresión estimado nos señala que el aumento de diplomados disminuye la Producción Científica del Grupo. La interpretación de este hecho se debe a que en esta línea los Grupos más productivos no suelen tener diplomados, mientras que los Grupos menos productivos son los que tienen más diplomados.

En todas las líneas se observa que en el modelo de regresión ajustado entre la Producción Científica y el número de doctores el incremento de doctores siempre incrementa la Producción Científica del Grupo. En cambio, en los modelos de regresión ajustados entre la Producción Científica y el tamaño del Grupo, se obtiene que la línea prioritaria de Ciencias de la Vida consigue que incrementos de tamaño también se incremente la Producción Científica; para el resto de las líneas prioritarias, los Grupos con tamaños muy grandes (última o penúltima categoría de la variable tamaño) disminuye la Producción Científica.

Por último, hemos elaborado un modelo de regresión múltiple entre la Producción Científica y las variables explicativas: Número de doctores, tamaño y cantidad concedida. En la tabla siguiente recogemos el coeficiente de correlación al cuadrado (en porcentajes) de dicho modelo, para cada una de las líneas prioritarias. 
TABLA 16

\begin{tabular}{lccccccc}
\hline & P.G.C. & A.A. & C.V. & R.N. & C.S. & T.P. & T.I. \\
\hline Doct., Tamaño y Cant. & 53 & 81 & 33 & 60 & 63 & 73 & 63 \\
\hline
\end{tabular}

FUENTE: Elaboración propia.

Se observa que para línea de Agroalimentación, las tres variables consideradas explican el $81 \%$ de la varianza de la Producción Científica. En cambio, para la línea de Ciencias de la Vida el porcentaje es del 33\%.

En la mayor parte de los modelos ajustados ente la Producción Científica y la tres variables explicativas, anteriormente seleccionadas, hemos encontrado que un incremento simultáneo en las tres variables explicativas hace que se incremente la producción del Grupo; haciendo notar que en alguno de los modelos ajustados la presencia de alta dependencia lineal entre las variables explicativas dificulta la interpretación de los resultados obtenidos (efecto de la colinealidad).

\section{CONCLUSIONES.}

A modo de resumen vamos a recoger en este apartado los aspectos que consideramos de mayor interés de los problemas abordados en nuestro trabajo.

a) La investigación en Andalucía está basada fundamentalmente en fondos aportados por el sector público.

b) La posición de Andalucía en publicación de documentos científicos en la base de datos SCI (está situada después de Madrid y Cataluña) no se corresponde con su posición en renta per cápita, (ocupa el penúltimo lugar en las 17 Comunidades Autónomas).

c) El impacto del Plan Nacional de investigación y el Plan Andaluz de Investigación en Andalucía ha sido importante para que los investigadores se organicen en Grupos de Investigación y que, por vez primera, se habla de la investigación como una tarea necesaria para el desarrollo de la región.

d) La implantación de un Sistema de Ciencia y Tecnología, nos lleva a la necesidad de desarrollar indicadores que permitan evaluar a las Instituciones, Departamentos, Grupos de Investigación e Investigadores. En nuestro trabajo indicamos que se debe hacer uso de la bibliometría tal como se viene empleando en otros países. Ahora bien, señalamos que se deben elaborar nuestras propias bases de datos como complemento a las bases de datos internacionales. La información aportada por la bibliometría debe, también, complementarse con información sobre fondos, recursos humanos, 
infraestructuras, relaciones entre ciencia y tecnología, etc. .., parte de esta información viene recogida en el apéndice del presente trabajo.

e) En nuestro trabajo hemos desarrollado un indicador sobre la Producción Científica de los Grupos de Investigación y estudiado posibles relaciones entre el indicador y características de cada Grupo. Señalamos la importancia del número de doctores como la variable más importante, para explicar la Producción Científica. También indicamos que los Grupos de grandes tamaños no significa que sean los grandes productores.

\section{BIBLIOGRAFÍA.}

ACOSTA, M. y CORONADO, D. (1991): Distribución Espacial y Políticas Regionales de I+D. XVII Reunión de Estudios Regionales. Barcelona.

BUSTO, J. (1991): Análisis de Correspondencia: Una Generalización para Datos Ordinales Multivariantes. Tesis Doctoral. Universidad de Sevilla.

GIFI, A. (1990): Nonlinear Multivariante Analysis. J. Wiley.

INE (1991): Estadísticas de Actividades de I+D. Madrid: INE.

LAFUENTE, A. y ORO, L. (1992): El sistema Español de Ciencias y Tecnología en el Marco Internacional: Evolución y perspectivas. FUNDESCO.

MALTRÁS, B. y QUINTANILLA, M. (1992): Producción Científica Española 1981-1989. Madrid: Consejo Superior de Investigaciones Científicas.

MARTÍN, C. y ROMERO, R. (1988): Datos para una política tecnológica de dimensión regional. Papeles de Economía Española, $n^{\circ}$ 35, páginas 153-164.

MARTÍN, C.; MORENO, L. y ROMERO, R. (1990): Estimación de la distribución regional de las actividades de I+D. Madrid: Fundación Empresa Pública, D.T. 9001.

P.A.I. (1990): Plan Andaluz de Investigación 1990-1993. Junta de Andalucía. Consejería de Educación y Ciencia.

RANIS, G. (1978): Science, Technology and Development: A restropective. Página 1-30 en G. Ranis, en Science, Technology and Economic Development 1978. Praeger.

SATO, R. y SUZOWA, G. (1983): Research and Productivity. Auburn.

SOLO, R. y ROGERS, E. (1972): Inducing Technical Change for Economic Growth and Development. Michigan: Michigan State Press University.

TISDELL, C.A. (1981): Science and Technology Policy. Cambridge: Cambridge University Press. SOLÍS CABRERA, F.; BASULTO, J. y ARIAS MARTÍN, C. (1992): Un estudio multivariante de los Grupos de Investigación inventariados por el P.A.I. en 1991. VI Reunión Anual de Assepelt-España. Granada. Páginas de 259-269.

SOLÍS CABRERA, F. y BASULTO, J. (1993): Posicionamiento de los Grupos de Investigación en Andalucía en función de la Productividad Científica: Propuesta de un indicador. VII Reunión Nacional de Assepelt-España. Cádiz. Páginas 386-398. 


\section{APÉNDICE ESTADÍSTICO.}

\begin{tabular}{lrrrrrrrr}
\hline $\begin{array}{l}\text { Public. Inter. } \\
\text { 1989 }\end{array}$ & P.G.C. & A.A. & C.V. & R.N. & C.S. & T.P. & T.I. & TOTAI \\
\hline U. Cádiz & 26 & 6 & 38 & 0 & 6 & 3 & 0 & 79 \\
U. Córdoba & 104 & 83 & 93 & 17 & 21 & 16 & 6 & 340 \\
U. Granada & 143 & 19 & 273 & 117 & 23 & 13 & 43 & 631 \\
U. Málaga & 55 & 3 & 91 & 43 & 34 & 40 & 13 & 279 \\
U. Sevilla & 99 & 10 & 221 & 23 & 111 & 84 & 37 & 585 \\
C.S.I.C. & 12 & 57 & 59 & 88 & 10 & 21 & 27 & 274 \\
Otros Órganos & 0 & 14 & 46 & 0 & 1 & 0 & 0 & 61 \\
Total & 439 & 192 & 821 & 288 & 206 & 177 & 126 & 2.249 \\
\hline
\end{tabular}

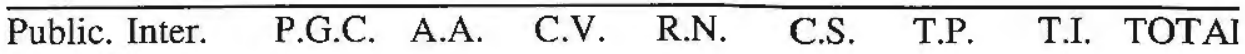
1990

\begin{tabular}{lrrrrrrrr}
\hline U. Cádiz & 26 & 5 & 53 & 6 & 4 & 15 & 0 & 109 \\
U. Córdoba & 106 & 118 & 88 & 16 & 18 & 14 & 6 & 366 \\
U. Granada & 151 & 23 & 365 & 131 & 13 & 18 & 38 & 739 \\
U. Málaga & 57 & 10 & 85 & 76 & 31 & 34 & 40 & 333 \\
U. Sevilla & 120 & 9 & 239 & 39 & 48 & 73 & 27 & 555 \\
C.S.I.C. & 12 & 67 & 63 & 77 & 4 & 31 & 49 & 303 \\
Otros Órganos & 0 & 13 & 46 & 0 & 1 & 0 & 0 & 60 \\
Total & 472 & 245 & 939 & 345 & 119 & 185 & 160 & 2.465 \\
\hline
\end{tabular}

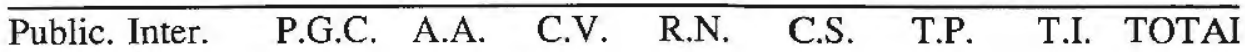
1991

\begin{tabular}{lrrrrrrrr}
\hline U. Cádiz & 27 & 1 & 28 & 9 & 10 & 21 & 0 & 96 \\
U. Córdoba & 70 & 136 & 107 & 13 & 38 & 23 & 4 & 391 \\
U. Granada & 183 & 30 & 337 & 160 & 47 & 11 & 51 & 819 \\
U. Málaga & 60 & 8 & 103 & 66 & 24 & 41 & 18 & 320 \\
U. Sevilla & 119 & 18 & 269 & 58 & 81 & 108 & 31 & 684 \\
C.S.I.C. & 13 & 58 & 73 & 92 & 9 & 26 & 29 & 300 \\
Otros Órganos & 2 & 11 & 38 & 0 & 3 & 0 & 0 & 54 \\
Total & 474 & 262 & 955 & 398 & 212 & -230 & 133 & 2.664 \\
\hline
\end{tabular}

FUENTE: Consejería de Educación y Ciencia. Junta de Andalucía 


\begin{tabular}{lrrrrrrrr}
\hline \begin{tabular}{l} 
Public. Nacio. \\
\multicolumn{1}{c}{ 1989 }
\end{tabular} & P.G.C. & A.A. & C.V. & R.N. & C.S. & T.P. & T.I. TOTAL \\
\hline U. Cádiz & 61 & 5 & 75 & 13 & 56 & 8 & 2 & 220 \\
U. Córdoba & 32 & 108 & 76 & 12 & 130 & 9 & 3 & 370 \\
U. Granada & 268 & 8 & 382 & 179 & 398 & 0 & 12 & 1.245 \\
U. Málaga & 67 & 0 & 63 & 45 & 222 & 28 & 38 & 463 \\
U. Sevilla & 199 & 20 & 227 & 53 & 455 & 37 & 12 & 1.003 \\
C.S.I.C. & 12 & 27 & 6 & 45 & 38 & 3 & 2 & 133 \\
Otros Órganos & 0 & 18 & 121 & 0 & 21 & 15 & 0 & 175 \\
Total & 639 & 186 & 950 & 347 & 1.320 & 100 & 69 & 3.611 \\
\hline
\end{tabular}

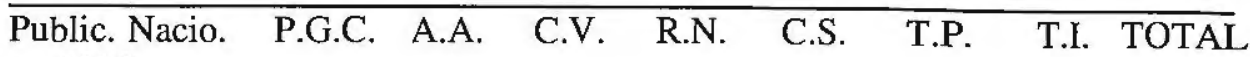
1990

\begin{tabular}{lrrrrrrrr}
\hline U. Cádiz & 43 & 11 & 116 & 1 & 59 & 9 & 0 & 239 \\
U. Córdoba & 22 & 120 & 54 & 23 & 133 & 5 & 3 & 360 \\
U. Granada & 213 & 2 & 375 & 163 & 426 & 0 & 6 & 1.185 \\
U. Málaga & 51 & 6 & 66 & 39 & 224 & 14 & 42 & 442 \\
U. Sevilla & 207 & 17 & 200 & 76 & 527 & 47 & 15 & 1.089 \\
C.S.I.C. & 6 & 36 & 5 & 46 & 28 & 2 & 8 & 131 \\
Otros Órganos & 0 & 12 & 133 & 0 & 38 & 9 & 0 & 192 \\
Total & 542 & 204 & 949 & 348 & 1.435 & 86 & 74 & 3.638 \\
\hline
\end{tabular}

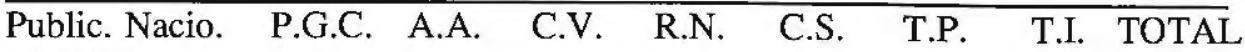
1991

\begin{tabular}{lrrrrrrrr}
\hline U. Cádiz & 84 & 13 & 72 & 13 & 71 & 8 & 0 & 261 \\
U. Córdoba & 64 & 103 & 55 & 10 & 171 & 8 & 2 & 413 \\
U. Granada & 283 & 24 & 302 & 162 & 363 & 4 & 6 & 1.144 \\
U. Málaga & 96 & 1 & 47 & 31 & 251 & 18 & 30 & 474 \\
U. Sevilla & 206 & 13 & 254 & 54 & 507 & 40 & 23 & 1.097 \\
C.S.I.C. & 5 & 41 & 7 & 43 & 24 & 0 & 2 & 122 \\
Otros Órganos & 14 & 6 & 98 & 0 & 9 & 0 & 0 & 127 \\
Total & 752 & 201 & 835 & 313 & 1396 & 78 & 63 & 3.638
\end{tabular}

FUENTE: Consejería de Educación y Ciencia. Junta de Andalucía 


\begin{tabular}{lrrrrrrrr}
\hline $\begin{array}{l}\text { Congr. Intern. } \\
\text { 1989 }\end{array}$ & P.G.C. & A.A. & C.V. & R.N. & C.S. & T.P. & T.I. & TOTA \\
\hline U. Cádiz & 41 & 0 & 59 & 5 & 5 & 11 & 0 & 121 \\
U. Córdoba & 44 & 83 & 88 & 10 & 26 & 3 & 4 & 258 \\
U. Granada & 98 & 32 & 450 & 87 & 48 & 13 & 19 & 747 \\
U. Málaga & 42 & 3 & 57 & 43 & 69 & 34 & 20 & 268 \\
U. Sevilla & 88 & 9 & 191 & 25 & 99 & 84 & 36 & 532 \\
C.S.I.C. & 8 & 65 & 35 & 48 & 21 & 16 & 29 & 222 \\
Otros Órganos & 0 & 7 & 58 & 0 & 4 & 0 & 0 & 69 \\
Total & 321 & 199 & 938 & 218 & 272 & 161 & 108 & 2.217 \\
\hline
\end{tabular}

\begin{tabular}{lrrrrrrrr}
\hline $\begin{array}{l}\text { Congr. Intern. } \\
\mathbf{1 9 9 0}\end{array}$ & P.G.C. & A.A. & C.V. & R.N. & C.S. & T.P. & T.I. & TOTAI \\
\hline U. Cádiz & 33 & 5 & 81 & 2 & 13 & 22 & 2 & 158 \\
U. Córdoba & 53 & 112 & 93 & 32 & 42 & 17 & 4 & 353 \\
U. Granada & 129 & 22 & 300 & 138 & 60 & 11 & 36 & 696 \\
U. Málaga & 20 & 4 & 91 & 52 & 74 & 39 & 16 & 296 \\
U. Sevilla & 120 & 20 & 185 & 15 & 144 & 88 & 43 & 615 \\
C.S.I.C. & 10 & 75 & 53 & 51 & 20 & 24 & 23 & 256 \\
Otros Órganos & 0 & 22 & 76 & 0 & 6 & 1 & 0 & 105 \\
Total & 365 & 260 & 879 & 290 & 359 & 202 & 124 & 2.479 \\
\hline
\end{tabular}

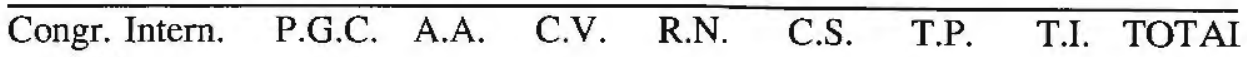
1991

\begin{tabular}{lrrrrrrrr}
\hline U. Cádiz & 15 & 2 & 57 & 29 & 14 & 49 & 0 & 166 \\
U. Córdoba & 32 & 139 & 121 & 11 & 51 & 8 & 9 & 371 \\
U. Granada & 155 & 52 & 362 & 105 & 133 & 15 & 43 & 865 \\
U. Málaga & 34 & 3 & 66 & 35 & 84 & 60 & 32 & 314 \\
U. Sevilla & 136 & 7 & 293 & 21 & 128 & 88 & 39 & 712 \\
C.S.I.C. & 13 & 74 & 75 & 63 & 13 & 18 & 31 & 287 \\
Otros Órganos & 1 & 11 & 103 & 4 & 4 & 0 & 0 & 123 \\
Total & 386 & 288 & 1.077 & 268 & 427 & 238 & 154 & 2.838 \\
\hline
\end{tabular}




\begin{tabular}{lrrrrrrrr}
\hline $\begin{array}{l}\text { Congr. Nacion. } \\
1989\end{array}$ & P.G.C. & A.A. & C.V. & R.N. & C.S. & T.P. & T.I. & TOTAL \\
\hline U. Cádiz & 40 & 12 & 169 & 2 & 22 & 11 & 0 & 256 \\
U. Córdoba & 47 & 47 & 146 & 3 & 61 & 3 & 5 & 312 \\
U. Granada & 176 & 23 & 581 & 107 & 150 & 1 & 8 & 1.046 \\
U. Málaga & 46 & 0 & 138 & 55 & 147 & 10 & 8 & 404 \\
U. Sevilla & 144 & 27 & 175 & 34 & 182 & 36 & 20 & 618 \\
C.S.I.C. & 1 & 40 & 42 & 40 & 12 & 11 & 0 & 146 \\
Otros Órganos & 0 & 6 & 208 & 0 & 4 & 3 & 0 & 221 \\
Total & 454 & 155 & 1.459 & 241 & 578 & 75 & 41 & 3.003 \\
\hline
\end{tabular}

\begin{tabular}{lllllll}
\hline Congr. Nacion. P.G.C. & A.A. & C.V. & R.N. & C.S. & T.P. & T.I. TOTAL
\end{tabular} 1990

\begin{tabular}{lrrrrrrrr}
\hline U. Cádiz & 34 & 4 & 163 & 7 & 26 & 18 & 2 & 254 \\
U. Córdoba & 30 & 85 & 177 & 18 & 66 & 8 & 6 & 390 \\
U. Granada & 163 & 5 & 516 & 125 & 199 & 3 & 19 & 1.030 \\
U. Málaga & 55 & 1 & 147 & 47 & 207 & 29 & 18 & 504 \\
U. Sevilla & 179 & 13 & 220 & 29 & 313 & 62 & 14 & 830 \\
C.S.I.C. & 1 & 67 & 25 & 36 & 17 & 15 & 12 & 173 \\
Otros Órganos & 0 & 12 & 268 & 0 & 5 & 2 & 0 & 287 \\
Total & 462 & 187 & 1.516 & 262 & 833 & 137 & 71 & 3.468 \\
\hline
\end{tabular}

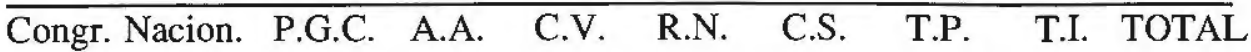
1991

\begin{tabular}{lrrrrrrrr}
\hline U. Cádiz & 44 & 6 & 109 & 28 & 46 & 12 & 0 & 245 \\
U. Córdoba & 34 & 110 & 114 & 22 & 111 & 12 & 15 & 418 \\
U. Granada & 134 & 41 & 579 & 131 & 187 & 8 & 34 & 1.114 \\
U. Málaga & 73 & 8 & 143 & 58 & 158 & 24 & 43 & 507 \\
U. Sevilla & 202 & 28 & 548 & 39 & 231 & 52 & 31 & 1.131 \\
C.S.I.C. & 70 & 46 & 28 & 32 & 19 & 7 & 0 & 139 \\
Otros Órganos & 18 & 12 & 225 & 0 & 5 & 0 & 0 & 260 \\
Total & 512 & 251 & 1.746 & 310 & 757 & 115 & 123 & 3.814 \\
\hline
\end{tabular}




\begin{tabular}{lrrrrrrrr}
\hline $\begin{array}{l}\text { Tesis Doctoral. } \\
1989\end{array}$ & P.G.C. & A.A. & C.V. & R.N. & C.S. & T.P. & T.I. & TOTAl \\
\hline U. Cádiz & 13 & 1 & 25 & 1 & 6 & 0 & 0 & 46 \\
U. Córdoba & 16 & 28 & 28 & 6 & 10 & 1 & 2 & 91 \\
U. Granada & 38 & 1 & 108 & 17 & 52 & 1 & 12 & 229 \\
U. Málaga & 18 & 1 & 31 & 10 & 19 & 3 & 9 & 91 \\
U. Sevilla & 37 & 9 & 69 & 10 & 62 & 8 & 8 & 203 \\
C.S.I.C. & 2 & 11 & 11 & 6 & 0 & 0 & 2 & 32 \\
Otros Órganos & 0 & 4 & 26 & 0 & 0 & 0 & 0 & 30 \\
Total & 124 & 55 & 298 & 50 & 149 & 13 & 33 & 722 \\
\hline
\end{tabular}

\begin{tabular}{lrrrrrrrr}
\hline $\begin{array}{l}\text { Tesis Doctoral. } \\
\quad \text { 1990 }\end{array}$ & P.G.C. & A.A. & C.V. & R.N. & C.S. & T.P. & T.I. & TOTAI \\
\hline U. Cádiz & 17 & 0 & 40 & 3 & 9 & 3 & 1 & 73 \\
U. Córdoba & 10 & 25 & 22 & 3 & 18 & 2 & 0 & 80 \\
U. Granada & 47 & 7 & 128 & 36 & 50 & 0 & 6 & 264 \\
U. Málaga & 21 & 1 & 25 & 8 & 27 & 4 & 9 & 95 \\
U. Sevilla & 54 & 8 & 90 & 14 & 72 & 8 & 6 & 252 \\
C.S.I.C. & 1 & 13 & 5 & 13 & 2 & 2 & 4 & 40 \\
Otros Órganos & 0 & 3 & 35 & 0 & 0 & 0 & 0 & 38 \\
Total & 150 & 57 & 345 & 67 & 178 & 19 & 26 & 842 \\
\hline
\end{tabular}

\begin{tabular}{|c|c|c|c|c|c|c|c|c|}
\hline $\begin{array}{c}\text { Tesis Doctoral } \\
1991\end{array}$ & P.G.C. & A.A. & C.V. & R.N. & C.S & T.P. & T.I. & TOTAI \\
\hline U. Cádiz & 14 & 0 & 26 & 2 & 9 & 7 & 0 & 58 \\
\hline U. Córdoba & 10 & 35 & 19 & 3 & 16 & 2 & 0 & 85 \\
\hline U. Granada & 60 & 7 & 99 & 21 & 62 & 2 & 10 & 261 \\
\hline U. Málaga & 19 & 3 & 16 & 14 & 41 & 3 & 7 & 103 \\
\hline U. Sevilla & 47 & 6 & 90 & 8 & 73 & 14 & 6 & 244 \\
\hline C.S.I.C. & 3 & 9 & 11 & 9 & 5 & 1 & 5 & 43 \\
\hline Otros Órganos & 10 & 5 & 27 & 0 & 0 & 0 & 0 & 42 \\
\hline Total & 163 & 65 & 288 & 57 & 206 & 29 & 28 & 836 \\
\hline
\end{tabular}




\begin{tabular}{lrrrrrrrr}
\hline \begin{tabular}{l} 
Proyectos \\
\multicolumn{1989}{c}{}
\end{tabular} & P.G.C. & A.A. & C.V. & R.N. & C.S. & T.P. & T.I. & TOTAL \\
\hline U. Cádiz & 8 & 9 & 26 & 10 & 7 & 6 & 1 & 67 \\
U. Córdoba & 18 & 47 & 31 & 18 & 18 & 4 & 12 & 148 \\
U. Granada & 36 & 4 & 83 & 91 & 50 & 4 & 20 & 288 \\
U. Málaga & 21 & 7 & 21 & 22 & 40 & 21 & 17 & 324 \\
U. Sevilla & 52 & 15 & 77 & 42 & 46 & 75 & 17 & 324 \\
C.S.I.C. & 1 & 39 & 25 & 40 & 11 & 8 & 13 & 137 \\
Otros Órganos & 0 & 21 & 34 & 0 & 3 & 2 & 0 & 60 \\
Total & 136 & 142 & 297 & 223 & 175 & 120 & 80 & 1.173 \\
\hline
\end{tabular}

\begin{tabular}{lrrrrrrrr}
\hline $\begin{array}{l}\text { Proyectos } \\
1990\end{array}$ & P.G.C. & A.A. & C.V. & R.N. & C.S. & T.P. & T.I. & TOTAL \\
\hline U. Cádiz & 6 & 1 & 36 & 10 & 9 & 10 & 2 & 74 \\
U. Córdoba & 12 & 58 & 31 & 14 & 23 & 3 & 10 & 151 \\
U. Granada & 44 & 7 & 103 & 709 & 70 & 5 & 26 & 364 \\
U. Málaga & 26 & 7 & 25 & 22 & 46 & 23 & 18 & 167 \\
U. Sevilla & 69 & 11 & 82 & 47 & 61 & 87 & 26 & 383 \\
C.S.I.C. & 1 & 45 & 30 & 50 & 8 & 11 & 14 & 159 \\
Otros Órganos & 0 & 23 & 38 & 0 & 1 & 2 & 0 & 64 \\
Total & 158 & 152 & 345 & 252 & 218 & 141 & 96 & 1.362 \\
\hline
\end{tabular}

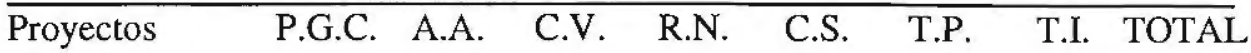
1991

\begin{tabular}{lrrrrrrrr}
\hline U. Cádiz & 17 & 14 & 36 & 2 & 16 & 22 & 2 & 108 \\
U. Córdoba & 18 & 80 & 31 & 13 & 25 & 6 & 12 & 185 \\
U. Granada & 67 & 13 & 137 & 97 & 96 & 8 & 43 & 461 \\
U. Málaga & 35 & 9 & 35 & 19 & 72 & 24 & 30 & 224 \\
U. Sevilla & 75 & 14 & 104 & 45 & 177 & 81 & 29 & 525 \\
C.S.I.C. & 11 & 58 & 44 & 39 & 17 & 14 & 12 & 195 \\
Otros Órganos & 1 & 9 & 31 & 0 & 9 & 0 & 0 & 50 \\
Total & 224 & 197 & 418 & 215 & 412 & 155 & 128 & 1.749 \\
\hline
\end{tabular}


\begin{tabular}{llllllll}
\hline Libros y Cap. de & P.G.C. & A.A. & C.V. & R.N. & C.S. & T.P. & T.I. TOTAI
\end{tabular} libros 1989

\begin{tabular}{lrrrrrrrr}
\hline U. Cádiz & 14 & 2 & 32 & 2 & 25 & 6 & 3 & 84 \\
U. Córdoba & 14 & 34 & 20 & 24 & 72 & 0 & 5 & 169 \\
U. Granada & 195 & 1 & 78 & 72 & 244 & 2 & 12 & 604 \\
U. Málaga & 40 & 0 & 21 & 32 & 161 & 3 & 13 & 720 \\
U. Sevilla & 87 & 17 & 48 & 36 & 304 & 227 & 12 & 731 \\
C.S.I.C. & 2 & 12 & 17 & 24 & 34 & 4 & 0 & 93 \\
Otros Órganos & 0 & 5 & 22 & 0 & 6 & 0 & 0 & 33 \\
Total & 352 & 71 & 238 & 190 & 846 & 242 & 45 & 1.984 \\
\hline
\end{tabular}

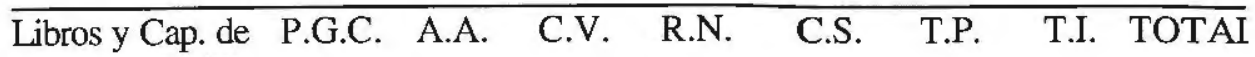
libros 1990

\begin{tabular}{lrrrrrrrr}
\hline U. Cádiz & 38 & 7 & 31 & 0 & 18 & 5 & 7 & 106 \\
U. Córdoba & 85 & 39 & 43 & 32 & 138 & 0 & 7 & 344 \\
U. Granada & 149 & 0 & 72 & 85 & 298 & 2 & 24 & 630 \\
U. Málaga & 52 & 0 & 34 & 24 & 245 & 6 & 18 & 379 \\
U. Sevilla & 119 & 4 & 54 & 31 & 457 & 22 & 18 & 705 \\
C.S.I.C. & 8 & 15 & 26 & 30 & 34 & 7 & 2 & 122 \\
Otros Órganos & 0 & 1 & 32 & 0 & 13 & 0 & 0 & 46 \\
Total & 451 & 66 & 292 & 202 & 1.203 & 42 & 76 & 1.332 \\
\hline
\end{tabular}

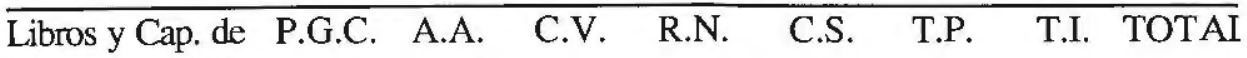
libros 1991

\begin{tabular}{lrrrrrrrr}
\hline U. Cádiz & 32 & 5 & 29 & 3 & 42 & 18 & 10 & 139 \\
U. Córdoba & 43 & 71 & 32 & 25 & 134 & 1 & 2 & 308 \\
U. Granada & 227 & 22 & 131 & 111 & 307 & 8 & 42 & 848 \\
U. Málaga & 97 & 2 & 16 & 25 & 224 & 9 & 9 & 382 \\
U. Sevilla & 109 & 9 & 69 & 30 & 470 & 68 & 13 & 768 \\
C.S.I.C. & 3 & 24 & 33 & 49 & 39 & 10 & 4 & 162 \\
Otros Órganos & 4 & 8 & 41 & 0 & 3 & 0 & 0 & 56 \\
Total & 515 & 141 & 351 & 243 & 1.219 & 114 & 80 & 2.663 \\
\hline
\end{tabular}

FUENTE: Consejería de Educación y Ciencia. Junta de Andalucía 